\title{
Spectral properties of a broad-area diode laser with off-axis external-cavity feedback
}

\section{Chi, Mingjun; Petersen, Paul Michael}

\section{Published in:}

Applied Physics Letters

Link to article, DOI:

$10.1063 / 1.4826613$

Publication date:

2013

Document Version

Publisher's PDF, also known as Version of record

Link back to DTU Orbit

Citation (APA):

Chi, M., \& Petersen, P. M. (2013). Spectral properties of a broad-area diode laser with off-axis external-cavity feedback. Applied Physics Letters, 103(17), [171112]. https://doi.org/10.1063/1.4826613

\section{General rights}

Copyright and moral rights for the publications made accessible in the public portal are retained by the authors and/or other copyright owners and it is a condition of accessing publications that users recognise and abide by the legal requirements associated with these rights.

- Users may download and print one copy of any publication from the public portal for the purpose of private study or research.

- You may not further distribute the material or use it for any profit-making activity or commercial gain

- You may freely distribute the URL identifying the publication in the public portal 


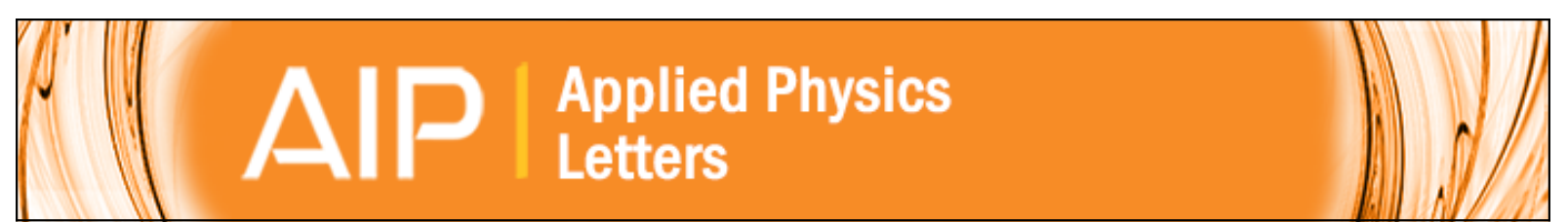

Spectral properties of a broad-area diode laser with off-axis external-cavity feedback Mingjun Chi and Paul Michael Petersen

Citation: Applied Physics Letters 103, 171112 (2013); doi: 10.1063/1.4826613

View online: http://dx.doi.org/10.1063/1.4826613

View Table of Contents: http://scitation.aip.org/content/aip/journal/apl/103/17?ver=pdfcov

Published by the AIP Publishing

Advertisement:

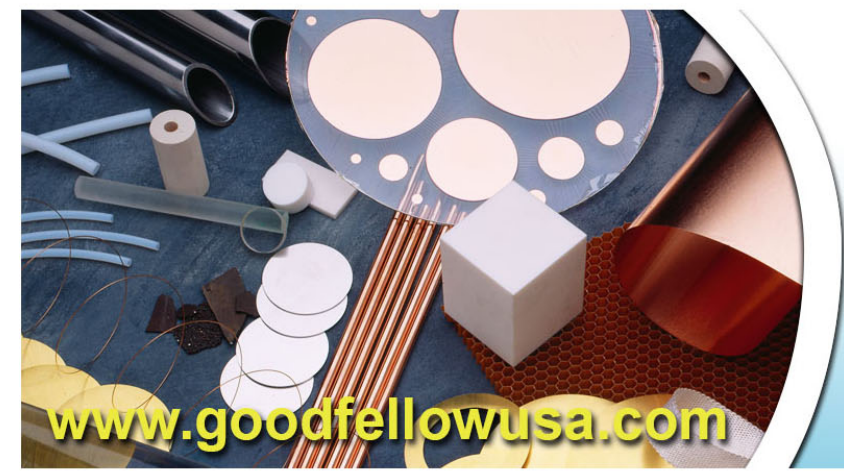

BODIFEl|OII

metals • ceramics $\cdot$ polymers composites • compounds • glasses

Save $5 \%$ B Buy online 70,000 products $\cdot$ Fast shipping 


\title{
Spectral properties of a broad-area diode laser with off-axis external-cavity feedback
}

\author{
Mingjun $\mathrm{Chi}^{\mathrm{a})}$ and Paul Michael Petersen \\ DTU Fotonik, Department of Photonics Engineering, Technical University of Denmark, Frederiksborgvej 399, \\ P.O. Box 49, DK-4000 Roskilde, Denmark
}

(Received 18 July 2013; accepted 10 October 2013; published online 23 October 2013)

\begin{abstract}
Spectral properties, both the optical spectrum and the intensity noise spectrum, of a broad-area diode laser with off-axis external-cavity feedback are presented. We show that the optical spectrum of the diode laser system is shifted to longer wavelengths due to the external-cavity feedback. The intensity noise spectrum of the diode laser shows that the intensity noise is increased strongly by the externalcavity feedback. External-cavity modes are excited in the external cavity even in the off-axis configuration. The peak spacing of the intensity noise spectrum shows that single roundtrip externalcavity modes are excited. We believe that the four-wave mixing process in the broad-area diode laser is responsible for the establishment of the external-cavity mode. (C) 2013 AIP Publishing LLC. [http://dx.doi.org/10.1063/1.4826613]
\end{abstract}

Broad-area diode lasers (BALs) suffer from poor spatial coherence owing to the broad emitter aperture in the slow axis, although they can produce high optical power and are attractive due to their compactness, long lifetimes, and low cost. Different techniques have been developed to improve the spatial coherence of BALs in the slow axis. Among them, off-axis external-cavity feedback is an effective technique to improve the beam quality of BALs in the slow axis. $^{1-8}$ The off-axis external-cavity feedback technique is based on the fact that the output of a gain-guided BAL consists of a set of transverse spatial modes; each mode has a double-lobed far-field in the slow axis, and these modes are distinguished at different angles in the far field. ${ }^{9,10}$ The offaxis external-cavity feedback technique is to select one lobe of a spatial mode at the far field and reflect it back into the laser cavity; thereby this lobe is amplified in the BAL and reflected at the back facet of the laser, finally it is coupled out of the laser as the other lobe (the output beam of the BAL system) of the same spatial mode. All the other transverse modes are suppressed effectively, ${ }^{1-8}$ thus, the spatial coherence of a BAL in the slow axis is improved significantly. But in order to achieve a compact laser system, normally for an off-axis external-cavity feedback set-up, the far field is imaged onto a pseudo-far-field plane by an imaging system, and different transverse spatial modes are distinguished at different positions in this plane, thus, the modeselection by angle in the real far field is changed to the mode-selection by position in the pseudo-far-field plane. ${ }^{1-4,6}$ The beam quality factor $M^{2}$ of the output beam of a BAL in slow axis can be improved up to 100 times by the off-axis external-cavity feedback technique. ${ }^{4}$

When an external-cavity feedback (both with a conventional mirror or a phase conjugate mirror) is applied to a semiconductor laser, both narrow-stripe ridge-waveguide laser $^{11,12}$ and BALs, ${ }^{13-19}$ external-cavity modes may be induced, and complex dynamics of the output beam from the laser system may take place. The spectral behavior and

${ }^{\text {a)} E l e c t r o n i c ~ m a i l: ~ m c h i @ f o t o n i k . d t u . d k ~}$ dynamics of BALs have been studied with short-cavity feedback, ${ }^{15,16,19}$ long-cavity feedback, ${ }^{14,17}$ and tilt mirror feedback, ${ }^{13,18}$ but all these kinds of feedbacks are not the off-axis external-cavity feedback as we described previously, i.e., no spatial mode selection is achieved in these research. ${ }^{13-19}$

Most of the BAL systems with off-axis external-cavity feedback are focused on the improvement of the spatial beam quality, i.e., to obtain a strongly asymmetric far field profile (or so-called single-lobe far field). ${ }^{1-8}$ Wolff et al. investigated the time-resolved lateral near- and far-field distribution of BAL with off-axis external-cavity feedback numerically, ${ }^{2}$ so far, the influence of the off-axis externalcavity feedback on the spectral properties and dynamics of a BAL has not been investigated experimentally. This influence is important when the BAL system is used for applications where the stability of the output beam is required.

In this Letter, we investigate the effect of the off-axis external-cavity feedback on the spectral properties of a BAL, both the optical spectrum and intensity noise spectrum are measured. Compared with the freely running condition, the optical spectrum of the BAL is shifted to the longer wavelength by the feedback, and the intensity noise spectrum is much stronger when the external-cavity feedback is applied. The peak spacing of the noise spectrum corresponds to a mode spacing of single roundtrip external-cavity modes. This means that stable single roundtrip longitudinal modes are established in the external-cavity.

The experimental setup is shown in Fig. 1. The BAL used in the experiment is an $810 \mathrm{~nm}, 3 \mathrm{~W}$ broad-area device with an emitter aperture of $1 \mu \mathrm{m} \times 200 \mu \mathrm{m}$ (fast $\times$ slow axis). The laser diode is antireflection coated on the front facet with a reflectivity of around $6 \%$, and high reflection coated on the back facet, the threshold current is around 0.6 A. The output beam is collimated in the fast axis by an aspherical lens of $4.5 \mathrm{~mm}$ focal length with a numerical aperture of 0.55 . Together with a cylindrical lens of $80 \mathrm{~mm}$ focal length, these two lenses collimate the beam in the slow axis and transform the near field to the far field at the pseudo-far-field plane simultaneously with respect to the slow axis. All lenses 


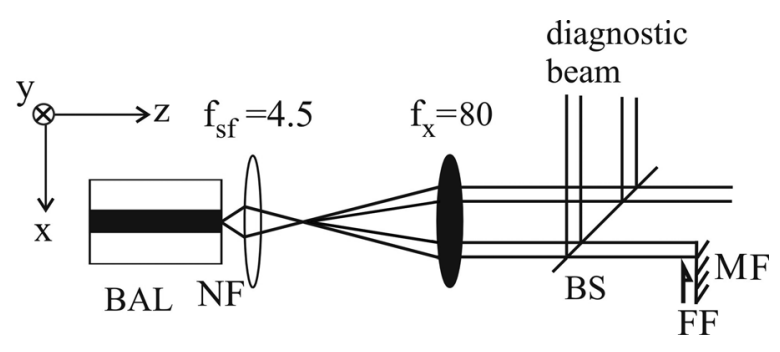

FIG. 1. Experimental setup of the BAL with off-axis external-cavity feedback; NF: Near field; BS: Beam splitter; FF: Far field; MF: Mirror with spatial filter.

are antireflection coated for near-infrared wavelength. At the pseudo-far-field plane, a mirror with a sharp edge combined with a razor blade is used as a mirror stripe to select one lobe of a transverse spatial mode and reflect it back into the gain medium of the BAL. The physical length of the external cavity is around $53 \mathrm{~cm}$. A beam splitter with a reflectivity of $1.2 \%$ is inserted into the external cavity, and the reflected beam is used as a diagnostic beam. The far-field profile, optical spectrum, and intensity noise spectrum of the output beam are measured on this beam.

The far-field profiles along the slow axis are measured with and without the external-cavity feedback at four different injected currents, and the results are shown in Fig. 2. The beam profiles are measured on the diagnostic beam by use of a beam scanner (Photon Inc., model 0180). At an operating current of $0.6 \mathrm{~A}$ (threshold of freely running BAL), as shown in Fig. 2(a), an one-main-lobe far-field profile is observed without the external-cavity feedback, this means mainly the zero-order transverse spatial mode oscillates on this condition. ${ }^{9,10}$ When the external-cavity feedback is applied, a very asymmetric far-field is obtained, and a strong lasing takes place (the threshold decreases to $0.56 \mathrm{~A}$ by the external feedback). The effect of the external feedback is a 2.5-times reduction in the output beam's width and an 11-times increase in the peak intensity compared with the freely running condition. As the operating current increases (up to
1.2 A, two times the threshold), more and more transverse spatial modes oscillate, thus, an $M$-shape far-field profile is observed without the feedback (as shown in Figs. 2(b)-2(d)), the far-field profile changes to a strongly asymmetric profile when the external-cavity feedback is applied. The effect of the external feedback with injected current high above the threshold is more than a 6-times reduction of the beam's width and a 4-times increase in the peak intensity compared with the freely running condition (as shown in Figs. 2(b)-2(d)).

The optical spectra of the output beam of the BAL with and without the external feedback are recorded by a spectrum analyzer (Advantest Corp., Q8347) at different injected currents and are shown in Fig. 3. Fig. 3(a) shows the optical spectra with an injected current of 0.6 A (threshold of the freely running $\mathrm{BAL}$ ), without the external feedback, only one longitudinal mode of the BAL oscillates, when the offaxis external-cavity feedback is applied, more longitudinal modes of the BAL oscillate simultaneously, and the spectrum is shifted to longer wavelength by the external feedback. When the injected current is high above the threshold (as shown in Figs. 3(b)-3(d)), more longitudinal modes of the BAL are induced in the laser cavity of the BAL with or without the external-cavity feedback, and the spectra are shifted to the longer wavelength.

When the off-axis external-cavity feedback is applied, the threshold of the BAL is decreased from 0.6 to $0.56 \mathrm{~A}$, and the output power of the BAL is increased around $30 \mathrm{~mW}$ accordingly with the four different injected currents. This means the carrier density in the active medium is decreased by the off-axis external-cavity feedback. Thus, the red shift of the spectrum of the BAL with external-cavity feedback may be caused by the band-filling effect.

The intensity noise spectrum of the output beam of our BAL system with and without the off-axis external-cavity feedback are monitored by a fast silicon PIN photodiode (Melles Griot, 13DAH001) connected with an electronic spectrum analyzer (band width $1.8 \mathrm{GHz}$, Tektronix 2753P),

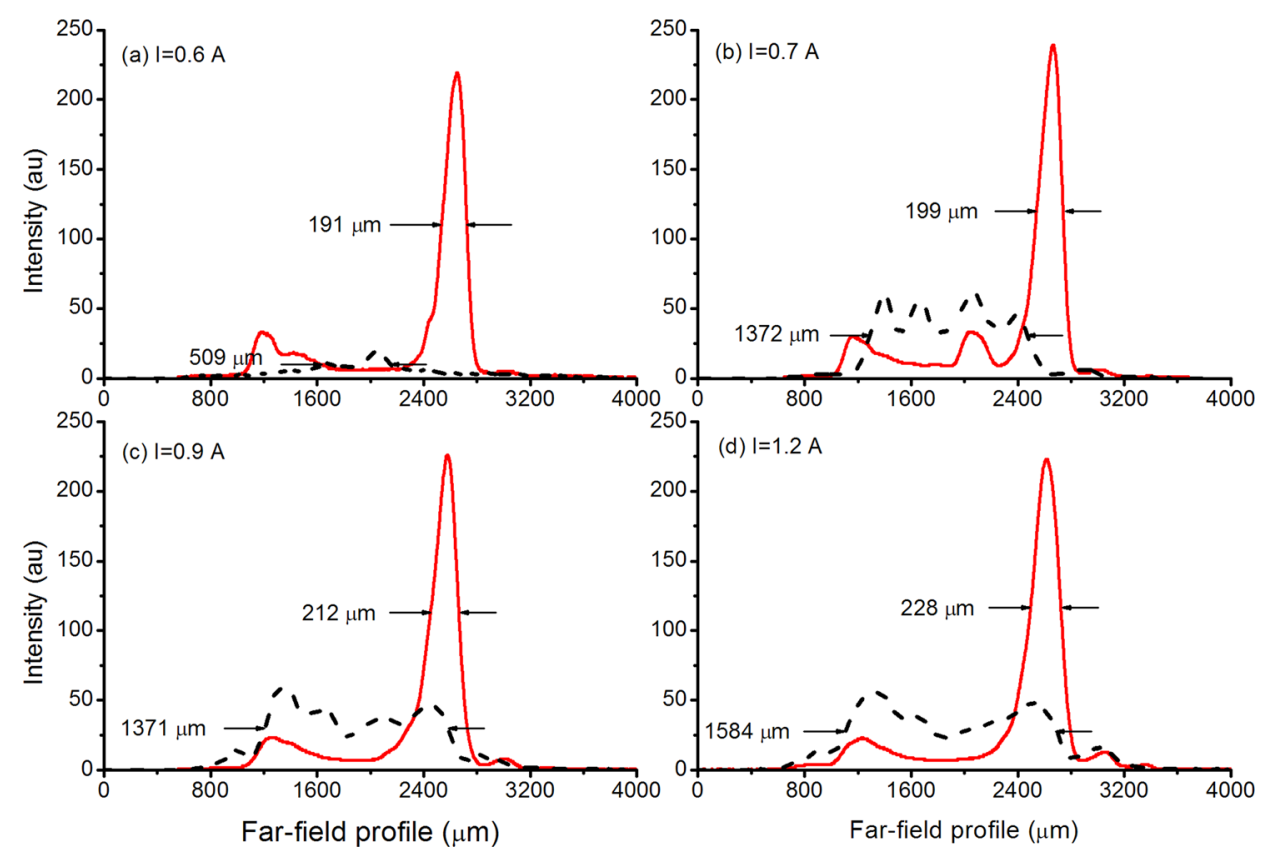

FIG. 2. Far-field profiles of the BAL measured along the slow axis without (black dashed curves) and with (red solid curves) the off-axis external-cavity feedback with injected currents of (a) $0.6 \mathrm{~A}$, (b) $0.7 \mathrm{~A}$, (c) $0.9 \mathrm{~A}$, and (d) $1.2 \mathrm{~A}$. 

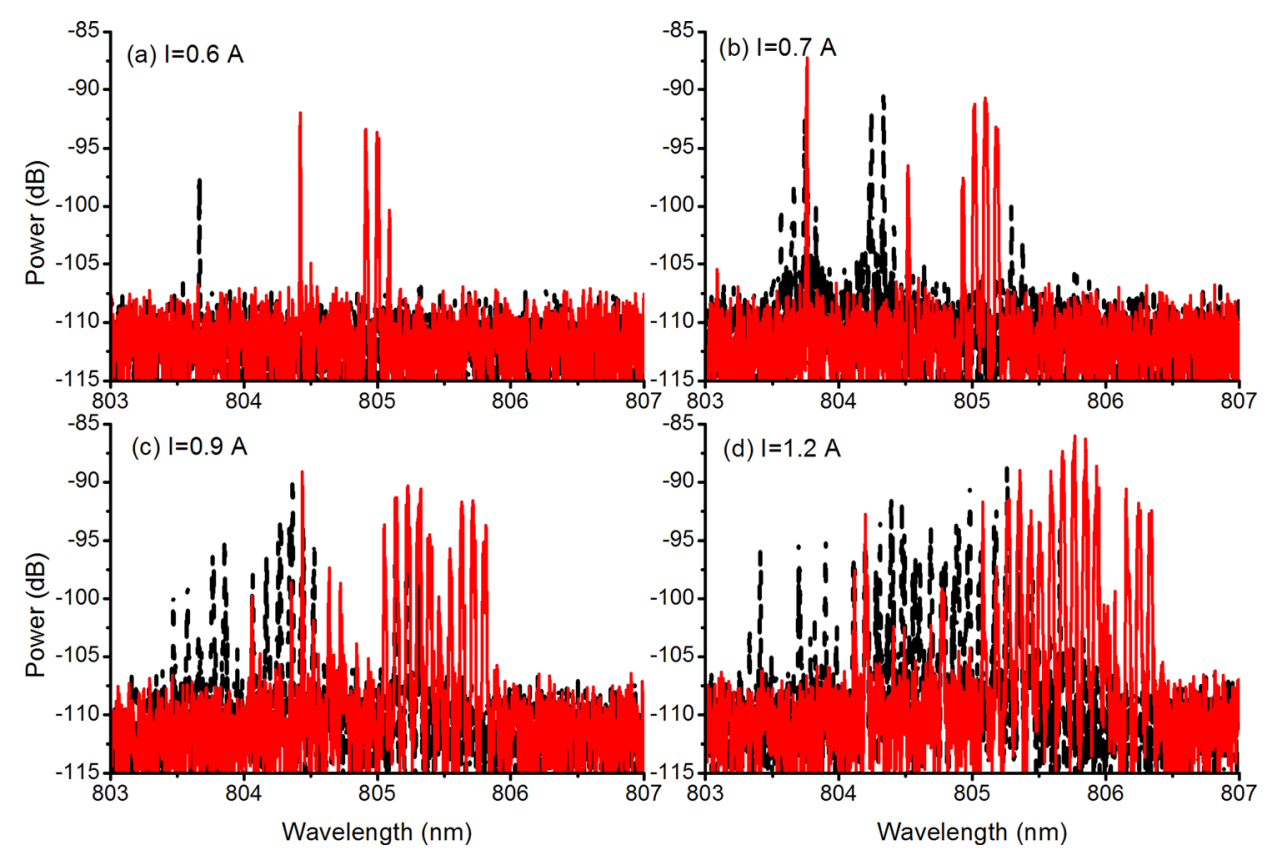

(d) $\mathrm{I}=1.2 \mathrm{~A}$
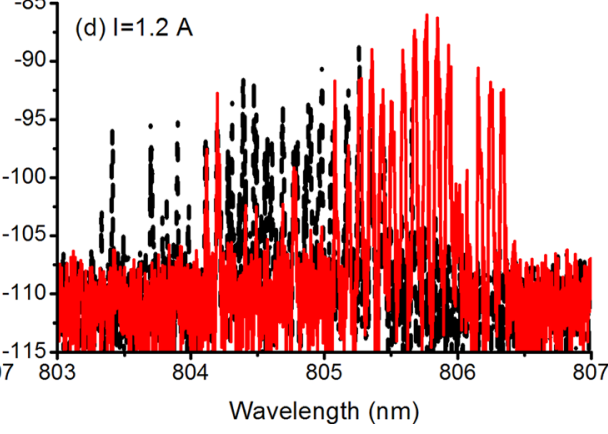

FIG. 3. Optical spectra of the output beam from the BAL without (black dashed curves) and with (red solid curves) the off-axis external-cavity feedback with injected currents of (a) $0.6 \mathrm{~A}$, (b) $0.7 \mathrm{~A}$, (c) $0.9 \mathrm{~A}$, and (d) $1.2 \mathrm{~A}$. and the spectra measured with four different injected currents are shown in Fig. 4.

Without the off-axis external-cavity feedback, the intensity noise is almost neglected in the measured noise spectrum range when the injected current is less than 0.9 A (Figs. 4(a)-4(c)), a small peak around $300 \mathrm{MHz}$ in the noise spectrum appears with injected current of $1.2 \mathrm{~A}$, and this is a resonance peak normally observed in both ridge-waveguide single-mode devices and BALs. ${ }^{20}$ When the external-cavity feedback is applied, the intensity noise is much stronger compared with the freely running condition, especially with high injected currents, the peaks of the intensity noise spectra are round $20 \mathrm{~dB}$ higher than the freely running condition. The peaks of the intensity noise spectra with spacing around $270 \mathrm{MHz}$ are originated from the beating between different external-cavity modes with the mode spacing of $270 \mathrm{MHz}$.
This means the external-cavity modes are excited in the external cavity even in the off-axis configuration.

The optical feedback strength is an important parameter for the research of dynamics in semiconductor laser. In order to obtain the best output beam quality, the position of the mirror stripe is different with different injected current, while the width of the mirror stripe is fixed. As the increase of the injected current, the output lobe is wider as seen in Fig. 2, this means more power is emitted in the non-selected modes. Thus, the feedback strength is decreased from $8.5 \%$ at $0.6 \mathrm{~A}$ to $7.0 \%$ at $1.2 \mathrm{~A}$, it is a moderate feedback strength for BAL. ${ }^{14}$ Figs. 3 and 4 show that the BAL system is operated in both multiple longitudinal modes of BAL and multiple external-cavity modes. It is similar to the conditions of regime IV in a narrow-striped diode $\operatorname{laser}^{12}$ or multimode unstable state in a $\mathrm{BAL}^{14}$ with a moderate optical feedback. (a)

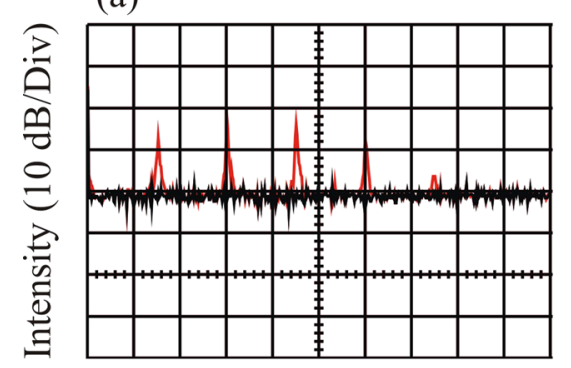

(c)

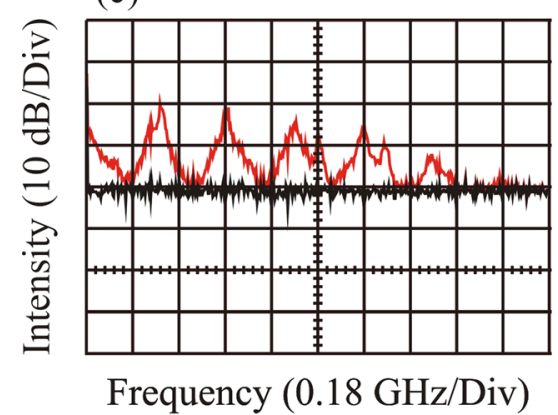

(b)

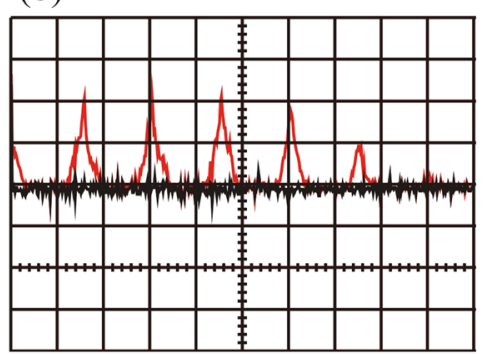

(d)

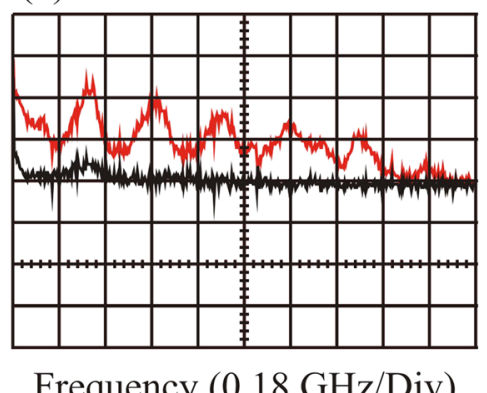

FIG. 4. Intensity noise spectra of the output beam from the BAL without (black curves) and with (red curves) the off-axis external-cavity feedback with injected currents of (a) $0.6 \mathrm{~A}$, (b) $0.7 \mathrm{~A}$, (c) $0.9 \mathrm{~A}$, and (d) $1.2 \mathrm{~A}$. 
For a conventional external-cavity feedback (mirror feedback), the optical field in the external cavity needs a single roundtrip to return to its initial state, thus, the dependence of the external-cavity mode spacing $\delta v$ on the cavity length (optical length) $L$ follows the relation: $\delta v=c / 2 L$, where $c$ is the speed of light. A $270 \mathrm{MHz}$ mode spacing in our case corresponds to a $55 \mathrm{~cm}$ optical length of the external cavity; this is in agreement with our cavity length of $53 \mathrm{~cm}$ when the refractive indices of the optical components are considered. Ostensibly, this implies that the external cavity in our experiment is a traditional resonator formed by a conventional feedback mirror and the end facet of the BAL. But if we check the off-axis external-cavity feedback configuration carefully, we find this is not the case for our experiment.

According to the principle of the off-axis external-cavity feedback presented previously, an equivalent laser resonator of the off-axis feedback BAL system is shown in Fig. 5(a), where $A_{4}$ is the lobe to the feedback mirror and reflected back by the mirror as $A_{3}$, afterwards $A_{3}$ is reflected by the back facet of the BAL as the output lobe $A_{1}$, and the beam is amplified during the propagation in the gain device. But in this process, the light reflected from the feedback mirror does not return to the external-cavity again, i.e., no laser oscillation is excited between the external mirror and the end facet of the BAL. Actually, this is normal since a stable resonator cannot be established between the feedback mirror and the end facet of the BAL due to the off-axis external-cavity feedback configuration. This is in disagreement with our experimental results of the existence of external-cavity modes.

We believe that a degenerate four-wave mixing (DFWM) process is responsible for the establishment of stable external-cavity mode in the off-axis feedback BAL system. An equivalent laser resonator of the BAL system including the DFWM is shown in Fig. 5(b); this model was

\section{(a)}

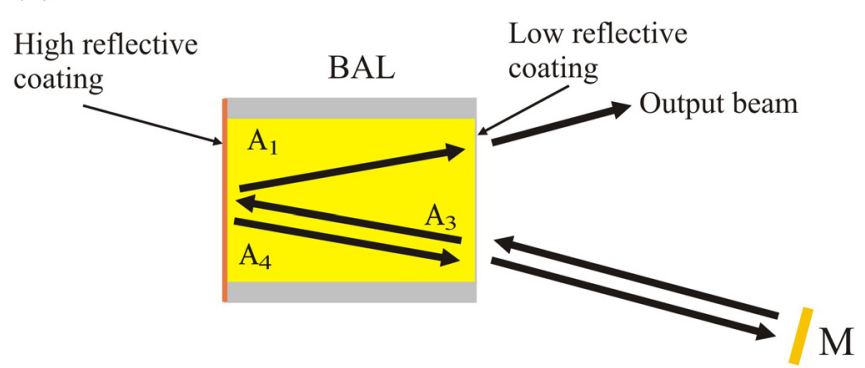

(b)

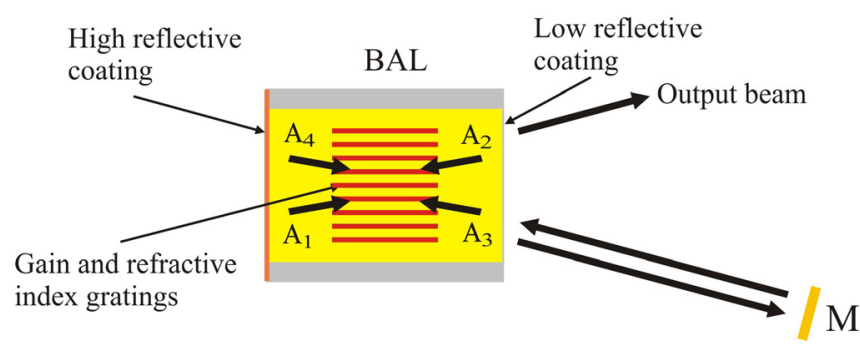

FIG. 5. The equivalent laser resonator of the BAL laser system with off-axis external-cavity feedback: (a) without and (b) with the phase conjugate mirror formed in the BAL by degenerate four-wave mixing; M: Feedback mirror. proposed previously to analyze the optimal feedback angle of a BAL with off-axis external-cavity feedback, ${ }^{21}$ but it focuses on the improvement of spatial beam quality, not the spectrum and dynamics process of a BAL with externalcavity feedback. Here, $A_{1}$ and $A_{4}$ are the output lobe and the lobe to the feedback mirror, an interference pattern is formed inside the gain medium, thus, a gain and a refractive index gratings are induced due to the spatial hole-burning effect and the anti-guiding effect. $A_{3}$ is the reflected lobe from the mirror, $A_{2}$ is generated by the diffraction on these gratings, and this is known as a degenerate four-wave mixing process; and the beam $A_{2}$ is reflected by the back facet of the BAL, and returns to the external cavity again, thus, a laser oscillation is excited. The existence of the gratings, i.e., the DFWM process, is the reason for the establishment of the external-cavity mode; otherwise the feedback beam $A_{3}$ will not return to the external-cavity, so no external-cavity mode can be excited.

Although the two output lobes from the BAL propagate off-axially in the off-axis external-cavity feedback BAL system, but the beam profile inside the BAL is an interference pattern of the four beams, thus, it is parallel to the $\mathrm{Z}$ direction in Fig. 1. The bright fringes of the pattern are called selfinduced "emitters" in Ref. 8, and a weak carrier population grating is created by "emitters"; 8 thus, a gain and a refractive index gratings are induced by the spatial hole-burning effect and anti-guiding effect, i.e., DFWM takes place inside the BAL. The existence of the interference pattern and these gratings (carrier density grating, gain, and refractive index gratings) are verified by the near-field profile of the BAL, the near-field profile with high intensity modulation is observed in both experimental results ${ }^{2-4,7,10}$ and theoretical investigation $^{2,5-8}$ on the off-axis external-cavity feedback system. Thus, the intensity modulated near-field profile is a proof of the occurrence of the DFWM process in the BAL with off-axis external-cavity feedback.

Theoretical study shows that for a resonator comprising a mirror and a phase-conjugate mirror induced by DFWM, stable external-cavity modes can be excited for both single and double roundtrips, i.e., with mode spacing of both $c / 2 L$ and $c / 4 L,{ }^{22}$ the mode that uses the gain most efficiently will be excited. In our experiment, the results show that the single roundtrip modes experience the gain more efficiently; this is the same as the case in Refs. 12 and 14.

In principle, when the external-cavity mode spacing is $c / 2 L$, it is impossible to distinguish that the cavity is formed by two ordinary mirrors or by an ordinary mirror and a phase conjugate mirror induced by DFWM. Our analysis above shows that a stable laser cavity cannot be established between the feedback mirror and the back facet of BAL due to the off-axis feedback configuration, thus, the external-cavity modes can only be excited in an externalcavity formed between the feedback mirror and a phase conjugate mirror induced by DFWM in the BAL; the intensity modulated near-field profile normally observed in a BAL with off-axis feedback verifies the existence of the gain and refractive index gratings due to the spatial holeburning effect and anti-guiding effect, i.e., the DFWM process takes place in the BAL to form the phase conjugate mirror. 
In conclusion, the spectrum properties of a BAL with off-axis external-cavity feedback are investigated. The optical spectrum of the BAL system is shifted to the longer wavelength due to the band filling effect. The intensity noise spectra of the output beam of the BAL system shows that the external-cavity modes are excited even in the off-axis configuration. Our analysis shows that the single roundtrip external-cavity modes are excited in the external cavity formed by the ordinary feedback mirror and the phaseconjugate mirror induced by DFWM in the BAL.

${ }^{1}$ C. J. Chang-Hasnain, J. Berger, D. R. Scifres, W. Streifer, J. R. Whinnery, and A. Dienes, Appl. Phys. Lett. 50, 1465 (1987).

${ }^{2}$ S. Wolff, A. Rodionov, V. E. Sherstobitov, and H. Fouckhardt, IEEE J. Quantum Electron. 39, 448 (2003).

${ }^{3}$ M. Chi, N.-S. B $ø$ gh, B. Thestrup, and P. M. Petersen, Appl. Phys. Lett. 85, 1107 (2004).

${ }^{4}$ M. Chi, B. Thestrup, and P. M. Petersen, Opt. Lett. 30, 1147 (2005).

${ }^{5}$ L. Lang, J. J. Lim, S. Sujecki, and E. C. Larkins, Opt. Quantum Electron. 40, 1097 (2008).

${ }^{6}$ J. J. Lim, S. Sujecki, L. Lang, Z. Zhang, D. Paboeuf, G. Pauliat, G. LucasLeclin, P. Georges, R. C. I. Mackenzie, P. Bream, S. Bull, K.-H. Hasler, B. Sumpf, H. Wenzel, G. Erbert, B. Thestrup, P. M. Petersen, N. Michel,
M. Krakowski, and E. C. Larkins, IEEE J. Sel. Top. Quantum Electron. 15, 993 (2009).

${ }^{7}$ A. Jechow, M. Lichtner, R. Menzel, M. Radziunas, D. Skoczowsky, and A. G. Vladimirov, Opt. Express 17, 19599 (2009).

${ }^{8}$ M. Lichtner, V. Z. Tronciu, and A. G. Vladimirov, IEEE J. Quantum Electron. 48, 353 (2012).

${ }^{9}$ J.-M. Verdiell and R. Frey, IEEE J. Quantum Electron. 26, 270 (1990).

${ }^{10}$ R. M. R. Pillai and E. M. Garmire, IEEE J. Quantum Electron. 32, 996 (1996).

${ }^{11}$ P. Kürz and T. Mukai, Opt. Lett. 21, 1369 (1996).

${ }^{12}$ J. S. Lawrence and D. M. Kane, Phys. Rev. A 63, 033805 (2001).

${ }^{13}$ P. P. Vasil'ev and I. H. White, Appl. Phys. Lett. 71, 40 (1997).

${ }^{14}$ J. S. Lawrence and D. M. Kane, J. Lightwave Technol. 20, 100 (2002).

${ }^{15}$ S. K. Mandre, I. Fischer, and W. Elsässer, Opt. Lett. 28, 1135 (2003).

${ }^{16}$ S. K. Mandre, I. Fischer, and W. Elsässer, Opt. Commun. 244, 355 (2005).

${ }^{17}$ Y. Fujita and J. Ohtsubo, Appl. Phys. Lett. 87, 031112 (2005).

${ }^{18}$ T. Tachikawa, R. Shogenji, and J. Ohtsubo, Opt. Rev. 16, 533 (2009).

${ }^{19}$ A. Takeda, R. Shogenji, and J. Ohtsubo, Appl. Phys. Lett. 101, 231105 (2012).

${ }^{20}$ Y. Lam, E. Espinosa, D. Nichols, L. Davis, and P. Bhattacharya, IEEE J. Quantum Electron. 29, 1018 (1993).

${ }^{21}$ P. M. Petersen, E. Samsøe, S. B. Jensen, and P. E. Andersen, Opt. Express 13, 3340 (2005).

${ }^{22}$ J. Auyeung, D. Fekete, D. M. Pepper, and A. Yariv, IEEE J. Quantum Electron. 15, 1180 (1979). 